Research Square
Preprints are preliminary reports that have not undergone peer review.

They should not be considered conclusive, used to inform clinical practice, or referenced by the media as validated information.

\title{
Self-Reported Mental Health During the COVID-19 Pandemic and its Association with Alcohol and Cannabis Use: A Latent Class Analysis
}

Nibene Habib Somé ( $\square$ Nibene.Some@camh.ca)

Centre for Addiction and Mental Health

Samantha Wells

Centre for Addiction and Mental Health

Daniel Felsky

Centre for Addiction and Mental Health

Hayley A. Hamilton

Centre for Addiction and Mental Health

Shehzad Ali

Western University

Tara Elton-Marshall

Centre for Addiction and Mental Health

Jürgen Rehm

Centre for Addiction and Mental Health

\section{Research Article}

Keywords: COVID-19, latent class analysis, mental health, alcohol, cannabis.

Posted Date: September 20th, 2021

DOI: https://doi.org/10.21203/rs.3.rs-828073/v1

License: (c) (i) This work is licensed under a Creative Commons Attribution 4.0 International License. Read Full License 


\section{Abstract}

Background: Mental health problems and substance use co-morbidities during the COVID-19 pandemic are a public health priority. Identifying individuals at high-risk of developing these problems can directly inform mitigating strategies. We aimed to identify distinct groups of individuals (i.e., latent classes) based on patterns of self-reported mental health symptoms and investigate associations with alcohol and cannabis use.

Methods: We used data from six successive waves of a web-based cross-sectional survey of adults aged 18 years and older living in Canada (6,021 participants). We applied latent class analysis to three domains of self-reported mental health: anxiety, depression, and loneliness. Logistic regression was used to characterize latent class membership, estimate the association of class membership with alcohol and cannabis use, and perform sex-based analyses.

Results: We identified two distinct classes: 1) individuals with low scores on all three mental health indicators (no/low-symptoms) and 2) those reporting high scores (high-symptoms). Those at greater risk of being in the high-symptoms class were likely to be women (adjusted odds ratio (aOR) $=1.34,95 \% \mathrm{Cl}: 1.18$ 1.52), people worried about getting COVID-19 ( $\mathrm{aOR}=2.39,95 \% \mathrm{Cl}: 2.02-2.82)$, and those with post-secondary education ( $\mathrm{aOR}=1.26,95 \% \mathrm{Cl}: 1.02-1.55)$. Asian ethnicity ( $\mathrm{aOR}=0.78,95 \% \mathrm{Cl}: 0.62-0.97)$, married status ( $\mathrm{aOR}=0.71,95 \% \mathrm{Cl}: 0.59-0.85)$, seniors ( $\mathrm{aOR}=0.38,95 \% \mathrm{Cl}: 0.32-0.47)$, individuals in households with income higher than CAD \$40,000: $\$ 40,000-\$ 79,000$ (aOR=0.73, 95\%Cl:0.60-0.90), \$80,000-\$119,000 (aOR=0.60, 95\%Cl:0.48-0.74) and \$120,000+ (aOR=0.47, $95 \% \mathrm{Cl}: 0.37-0.59)$ were at lower odds of being in the high-symptoms class. Individuals in the high-symptoms class were more likely to use cannabis at least once a week (aOR=2.25, 95\%Cl:1.90-2.67), drink alcohol heavily ( $\mathrm{aOR}=1.69,95 \% \mathrm{Cl}: 1.47-1.95)$; and increase the use of cannabis (aOR=3.48, 95\%Cl:2.79-4.35) and alcohol (aOR=2.37, 95\% Cl:2.05-2.73) during the pandemic. Women in the high-symptoms class had higher odds of increasing alcohol use than men.

Conclusions: We identified the determinants of experiencing high-symptoms of anxiety, depression, and loneliness, and found a significant association with alcohol and cannabis consumption. This suggests that initiatives and supports are needed to address mental health and substance use multi-morbidities, particularly regarding alcohol use in women.

\section{Introduction}

Evidence indicates that the COVID-19 pandemic and related public health directives have led to elevated mental health symptoms, including depression, loneliness, and anxiety among individuals worldwide (1-3). Evidence also shows that social isolation and loneliness, key problems in the context of lockdown orders in effect due to the pandemic, are associated with depression and anxiety disorder (4-6). A review of studies on the mental health impact of the pandemic reported that between $16 \%$ and $28 \%$ of individuals have reported symptoms of anxiety and depression (7). Another systematic review reported relatively high rates of symptoms of anxiety (6-51\%) and depression (15-48\%) (8). These studies, though important for reporting prevalence rates, lack data on patterns of responses across mental health conditions. Thus, relatively little is known about how these mental health symptoms may cluster together.

Depression and anxiety are often paired together (9-11). Of people with a diagnosis of depression, $67 \%$ had a current and $75 \%$ had a lifetime co-morbid anxiety disorder (11). Similarly, of those experiencing anxiety, $63 \%$ had a current and $81 \%$ had a lifetime depressive disorder (11). Recent studies show that loneliness predicts higher depression and anxiety symptoms,(12) that there is a positive association between loneliness due to the COVID-19 pandemic and anxiety and depressive symptoms,(13) and that loneliness increases the odds for depression, anxiety, and their co-morbidity during the pandemic (14). Since mental health symptoms frequently coexist in people, and risk indicators for co-morbid disorders might differ from those experiencing single disorders, $(15,16)$ it is important to simultaneously examine mental health symptoms to unveil patterns of co-occurrence and association with substance use. This can be achieved with latent class analysis (LCA), a statistical method that creates groups of individuals with similar patterns of characteristics referred to as latent classes (17). LCA is recognized as a useful tool for studying and classifying mental health disorders at the population level (18). Identifying these classes is important in identifying distinct patterns to determine how mental health disorders cluster together (i.e., class membership) and investigate whether classes change over time and by sex. The identification of groups of individuals with mental health multi-morbidities has important implications for public health policy (resource allocation, raising awareness, or/and further screening) because the presence of multi-morbidity reduces quality of life (19, 20). In addition, it may inform the design of interventions or adapt existing interventions to meet the needs of people with multi-morbidities, particularly when they are at risk of substance abuse.

Evidence shows that mental illness, including symptoms of depression and anxiety, is positively associated with both alcohol (21-23) and cannabis use (2426). Recent research has shown that individuals consumed more alcohol $(27-30)$ and more cannabis $(27,31,32)$ than before the pandemic, with experts suggesting that people may be using alcohol and cannabis to cope with the stressors experienced during the pandemic with important sex differences (2, 33). Alcohol and cannabis use may potentially weaken the immune response in regards to infections (34-39). Thus, it is important to investigate the association between mental health symptoms, and latent class membership, with individuals' substance use behavior during the pandemic. Of particular interest is whether groups of individuals reporting high levels of anxiety, depression, and/or loneliness are more likely to use alcohol and/or cannabis. Such investigations may help improve understanding of mental health and substance use co-morbidities and multi-morbidities during the pandemic to inform and support actions that promote mental health and prevent substance use problems.

We used data from a series of surveys in Canada to: (1) examine latent classes of participants on indicator variables of anxiety, depression, and loneliness during the COVID-19 pandemic using LCA; (2) explore risk factors associated with latent class membership; and (3) estimate the associations of latent class membership with alcohol and cannabis consumption during the COVID-19 pandemic, test the associations for sex and time differences.

\section{Methods}

\section{Study design and participants}


This study used data from six successive waves of web-based cross-sectional Canada-wide surveys of adults aged 18 years and older. The surveys were conducted in English by the firm Delvinia. The sample was derived from a web-based survey panel, and quota sampling was used to approximate the distribution of the English-speaking Canadian population by age, sex, and region (40). Electronic informed consent was obtained prior to initiating the survey. The study received ethics approval from the Centre for Addiction and Mental Health. The surveys were conducted in six waves in 2020 as follows: May 8-12 (Wave 1, $n=1,005$, response rate (RR) $=15.9 \%$ ), May 29-June 1 (Wave 2, $n=1,002$, RR = 17.2\%), June 19-23 (Wave 3, $n=1,005, R R=16.4 \%)$, July $10-14$ (Wave $4, n=1,003, R R=13.7 \%$ ), September 18-22 (Wave 5, $n=1,003, R R=17.6 \%$ ), and November 27-December 1 (Wave $6, n=1,003, R R=16.2 \%$ ). The details of the survey interviews information and RR calculations are in Table A.1 of the Appendix. A pooled sample of 6,021 participants (Waves 1-6) was analyzed in this study. These data were collected at different points to permit an examination of variation in the impact of COVID-19-related stressors on participants over time.

\section{Measures}

\section{Mental health indicators}

We identified anxiety among participants using the 7-item generalized anxiety disorder, using the GAD-7 scale, which is based on 4-point Likert-scale questions. These items measure the frequency of anxiety symptoms over the past two weeks and are scored from 0 (not at all) to 3 (nearly every day). The summary score ranged from 0 to 21 (41). A score $\geq 10$ suggests moderate or severe anxiety to consider treatment, (42) which has clinical relevance. The literature that have studied GAD-7 scale have also validated the cut-off of 10 (42-44). We then constructed a binary variable for anxiety to identify participants with moderate or severe anxiety symptoms (45).

Participants who felt depressed were identified using a question from the Center for Epidemiologic Studies Depression Scale (CES-D) (46): "In the past 7 days, how often have you felt depressed?" Response options included: "rarely or none of the time (less than 1 day)", "some or a little of the time (1-2 days)", "occasionally or a moderate amount of the time (3-4 days)", and "most or all of the time (5-7 days)". Participants who reported feeling depressed 3-4 or more days in the previous week were classified as experiencing depressive symptoms (46). Similarly, loneliness was measured with a single item from the CES-D (46) with the same response options: "In the past 7 days, how often have you felt lonely?" Participants were considered to be lonely if they reported feeling lonely for 3-4 or more days in the previous week (46).

Although LCA is a data-driven method, extra steps are needed to ensure that identified classes are interpretable and not simply statistical artefacts (47). We described the classes and determined the factors that are associated with the classes.

\section{Alcohol and cannabis use variables}

Four variables related to alcohol and cannabis use were assessed. For alcohol, a binary variable identifying heavy episodic drinkers was derived based on the responses to the question: "On how many of the past seven days did you drink four (if woman) or five (if man) or more drinks on one occasion?" Men who consumed five (four for women) or more drinks per occasion at least four days per week were coded as heavy episodic drinkers. Note that a drink was defined as a $12 \mathrm{oz}$. bottle of beer or cider/cooler ( $5 \%$ alcohol content), a 5 oz. glass of wine (12\% alcohol content), or a straight or mixed drink with 1.5 oz. of liquor (40\% alcohol content). The second alcohol use question examined whether people's drinking increased due to the pandemic. Participants were asked: "In the past seven days, did you drink more alcohol, about the same or less alcohol overall than you did before the COVID-19 pandemic started?" This measure was coded to reflect an increase in alcohol use as: 0 (much less, slightly less, or same), and 1 (slightly more or much more).

For cannabis use, participants were asked: "During the past seven days, on how many days did you use cannabis?" A binary measure was created to reflect any cannabis use (use on one or more days) versus no cannabis use in the past week. Increase in cannabis use was also measured with the question: "In the past 7 days, did you use cannabis more often, about the same, or less often overall than you did before the COVID-19 pandemic started?" This was coded to reflect an increase in cannabis use as follows: 0 (much less, slightly less, or same), and 1 (slightly more or much more).

\section{Covariates}

We included several individual and household covariates: sex, age (18-39, 40-59 and 60 years or more), marital status (married/living with partner, separated/divorced/widowed and single), educational status (high school or less, some post-secondary, college degree/diploma and university degree/diploma), racial group (White, Asian, Black/Indigenous/Arab/Latinos and other ethnicities), residential environment (urban, suburban and rural), household income (less than $\$ 40,000, \$ 40,000-\$ 79,999, \$ 80,000-\$ 119,999, \$ 120,000$ or more, and 'prefer not to answer'), having children under 18 in the household and household composition (living alone or living with others). We also included a variable indicating the extent of worry experienced regarding contracting COVID-19, based on responses to the question: "How worried are you that you or someone close to you will get ill from COVID-19?", with possible responses provided on a 4-point Likert scale of: "very worried", "somewhat worried", "not very worried", and "not at all worried". We derived a binary variable to compare those classified as worried (i.e., very or somewhat worried) versus those not worried (i.e., not very or not at all worried). We accounted for time effects by adding a binary variable for each wave.

\section{Statistical analyses}

We used LCA to identify classes of participants with similar patterns of reported mental health symptoms during the pandemic. We used the three mental health indicators (anxiety, depression, loneliness) to divide participants into mutually exclusive and exhaustive latent classes. Using LCA, we estimated the probability for each participant of being in a particular class based on their responses to all three indicator items. We used the gsem command in Stata to fit the latent class model.

To determine the optimal number of latent classes, we estimated latent class models using different class numbers and we used the Akaike's (AIC) and the Bayesian information criterion (BIC) to select the model with the better fit.(48) 
To assess the associations of latent class memberships with alcohol and cannabis use, we used multivariate logistic regression. We adjusted for individual participant confounders (sex, age, education, marital status, ethnicity, residential environment), household confounders (income, presence of children, presence of other persons in the home), worrying about getting COVID-19, and survey wave indicator variables. We tested for sex differences by including latent class by sex interactions. We also included latent class by wave interaction terms to assess whether and how the association of class membership with alcohol and cannabis use changed over time. We then calculated the F-test for the joint significance of interaction terms to detect time/wave effects. We also regressed anxiety, depression and loneliness variables on alcohol and cannabis use to assess the association of individual mental health symptoms on alcohol and cannabis use.

We presented descriptive statistics of the cohort, including percentages and number of observations. We also reported adjusted odds ratios (aORs) with $95 \%$ confidence intervals (Cls), and presented results by sex. We used Stata (version 16.0) for all analyses. The full estimation tables are in the Appendix (Table A.2-3).

\section{Results}

A total of 6,021 participants completed the survey across the six waves, with at least 1,000 participants per wave. In Table 1, we report the number and the percentage of participants for each self-reported measure of mental health symptom, alcohol and cannabis use, as well as participants' characteristics within each wave, and for the total sample (all six waves). Overall, the percentage of participants who reported severe/moderate anxiety, depression, and loneliness were quite similar across the waves and in the full sample (ranging between $19-25 \%, 18-22 \%$, and $20-24 \%$, respectively). Between $12-16 \%$ of participants reported using cannabis at least once a week, and $24-27 \%$ reported engaging in heavy episodic drinking. Regarding change in cannabis and alcohol use, a total of 401 (7\%) and 1,295 (22\%) participants reported having increased their use of cannabis and alcohol.

\section{Latent class modeling and identification of classes}

Models with one to four latent classes for each wave and for pooled waves were estimated and compared using the information criteria (see Table A.4 in the Appendix). All three criteria (Log-likelihood, AIC and BIC) indicated that the two-class models resulted in a better fit than other models. However, we also characterized used the three-class model and estimated the association between three-latent class variable and substance use to assess sensitivity of the results to the number of class (results for the three-class model are in the Table A.5-6 in Appendix). 
Table 1

Descriptive statistics: Mental health indicators, substance use and sociodemographic characteristics

\begin{tabular}{|c|c|c|c|c|c|c|c|c|c|c|c|c|c|c|}
\hline \multirow[b]{2}{*}{ Variables } & \multicolumn{2}{|c|}{ Wave 1} & \multicolumn{2}{|c|}{ Wave 2} & \multicolumn{2}{|c|}{ Wave 3} & \multicolumn{2}{|c|}{ Wave 4} & \multicolumn{2}{|c|}{ Wave 5} & \multicolumn{2}{|c|}{ Wave 6} & \multicolumn{2}{|c|}{ All waves } \\
\hline & $\mathrm{n}$ & $\%$ & $\mathrm{n}$ & $\%$ & $\mathrm{n}$ & $\%$ & $\mathrm{n}$ & $\%$ & $\mathrm{n}$ & $\%$ & $\mathrm{n}$ & $\%$ & $\mathrm{n}$ & $\%$ \\
\hline \multicolumn{15}{|l|}{ Mental health indicators } \\
\hline Moderate/severe anxiety & 256 & $25.5 \%$ & 215 & $21.5 \%$ & 196 & $19.5 \%$ & 193 & $19.2 \%$ & 212 & $21.1 \%$ & 244 & $24.3 \%$ & 1319 & $21.9 \%$ \\
\hline Felt depressed & 205 & $20.4 \%$ & 212 & $21.2 \%$ & 185 & $18.4 \%$ & 188 & $18.7 \%$ & 213 & $21.2 \%$ & 218 & $21.7 \%$ & 1222 & $20.3 \%$ \\
\hline Felt lonely & 233 & $23.2 \%$ & 237 & $23.7 \%$ & 211 & $21.0 \%$ & 231 & $23.0 \%$ & 202 & $20.1 \%$ & 234 & $23.3 \%$ & 1349 & $22.4 \%$ \\
\hline \multicolumn{15}{|l|}{ Alcohol and cannabis use } \\
\hline Cannabis use past week & 115 & $11.5 \%$ & 130 & $13.0 \%$ & 124 & $12.4 \%$ & 131 & $13.1 \%$ & 119 & $11.9 \%$ & 160 & $16.0 \%$ & 781 & $13.0 \%$ \\
\hline Heavy Episodic Drinking & 238 & $23.7 \%$ & 247 & $24.7 \%$ & 267 & $26.6 \%$ & 271 & $27.2 \%$ & 255 & $25.5 \%$ & 257 & $25.7 \%$ & 1537 & $25.6 \%$ \\
\hline Increase in cannabis use & 64 & $6.4 \%$ & 70 & $7.0 \%$ & 59 & $5.9 \%$ & 62 & $6.2 \%$ & 53 & $5.3 \%$ & 93 & $9.3 \%$ & 401 & $6.7 \%$ \\
\hline Increase in alcohol use & 253 & $25.2 \%$ & 244 & $24.4 \%$ & 216 & $21.5 \%$ & 209 & $20.8 \%$ & 168 & $16.7 \%$ & 208 & $20.7 \%$ & 1295 & $21.5 \%$ \\
\hline \multicolumn{15}{|l|}{ Covariates } \\
\hline Men & 504 & $50.1 \%$ & 492 & $49.1 \%$ & 501 & $49.9 \%$ & 502 & $50.0 \%$ & 497 & $49.6 \%$ & 492 & $49.1 \%$ & 2986 & $49.6 \%$ \\
\hline Women & 498 & $49.6 \%$ & 497 & $49.6 \%$ & 499 & $49.7 \%$ & 492 & $49.1 \%$ & 498 & $49.7 \%$ & 503 & $50.1 \%$ & 2986 & $49.6 \%$ \\
\hline Living with others & 797 & $79.5 \%$ & 788 & $78.9 \%$ & 787 & $78.7 \%$ & 799 & $79.9 \%$ & 797 & $79.6 \%$ & 796 & $79.8 \%$ & 4763 & $79.4 \%$ \\
\hline Living alone & 205 & $20.5 \%$ & 211 & $21.1 \%$ & 213 & $21.3 \%$ & 201 & $20.1 \%$ & 204 & $20.4 \%$ & 201 & $20.2 \%$ & 1236 & $20.6 \%$ \\
\hline Presence of children & 229 & $22.8 \%$ & 236 & $23.6 \%$ & 237 & $23.6 \%$ & 242 & $24.1 \%$ & 234 & $23.3 \%$ & 216 & $21.5 \%$ & 1397 & $23.2 \%$ \\
\hline No children & 776 & $77.2 \%$ & 766 & $76.4 \%$ & 768 & $76.4 \%$ & 761 & $75.9 \%$ & 769 & $76.7 \%$ & 787 & $78.5 \%$ & 4624 & $76.8 \%$ \\
\hline Income less than $\$ 40 \mathrm{~K}$ & 128 & $12.7 \%$ & 121 & $12.1 \%$ & 136 & $13.5 \%$ & 118 & $11.8 \%$ & 116 & $11.6 \%$ & 110 & $11.0 \%$ & 729 & $12.1 \%$ \\
\hline Income in $\$ 40,000-\$ 79,999$ & 268 & $26.7 \%$ & 236 & $23.6 \%$ & 238 & $23.7 \%$ & 235 & $23.4 \%$ & 247 & $24.6 \%$ & 236 & $23.5 \%$ & 1457 & $24.2 \%$ \\
\hline Income in $\$ 80,000-\$ 119,999$ & 226 & $22.5 \%$ & 229 & $22.9 \%$ & 220 & $21.9 \%$ & 213 & $21.2 \%$ & 237 & $23.6 \%$ & 241 & $24.0 \%$ & 1367 & $22.7 \%$ \\
\hline Income $\$ 120,000+$ & 217 & $21.6 \%$ & 259 & $25.8 \%$ & 247 & $24.6 \%$ & 252 & $25.1 \%$ & 228 & $22.7 \%$ & 251 & $25.0 \%$ & 1451 & $24.1 \%$ \\
\hline Income missing & 166 & $16.5 \%$ & 157 & $15.7 \%$ & 164 & $16.3 \%$ & 185 & $18.4 \%$ & 175 & $17.4 \%$ & 165 & $16.5 \%$ & 1012 & $16.8 \%$ \\
\hline College & 190 & $18.9 \%$ & 211 & $21.1 \%$ & 189 & $18.8 \%$ & 204 & $20.3 \%$ & 183 & $18.2 \%$ & 221 & $22.0 \%$ & 1198 & $19.9 \%$ \\
\hline High school & 111 & $11.0 \%$ & 104 & $10.4 \%$ & 129 & $12.8 \%$ & 122 & $12.2 \%$ & 119 & $11.9 \%$ & 99 & $9.9 \%$ & 686 & $11.4 \%$ \\
\hline Post-secondary & 159 & $15.8 \%$ & 165 & $16.5 \%$ & 148 & $14.7 \%$ & 162 & $16.2 \%$ & 147 & $14.7 \%$ & 150 & $15.0 \%$ & 933 & $15.5 \%$ \\
\hline University & 538 & $53.5 \%$ & 516 & $51.5 \%$ & 531 & $52.8 \%$ & 502 & $50.0 \%$ & 548 & $54.6 \%$ & 521 & $51.9 \%$ & 3155 & $52.4 \%$ \\
\hline Asian & 200 & $19.9 \%$ & 175 & $17.5 \%$ & 201 & $20.0 \%$ & 188 & $18.7 \%$ & 190 & $18.9 \%$ & 202 & $20.1 \%$ & 1156 & $19.2 \%$ \\
\hline $\begin{array}{l}\text { Black/Indigenous/Arab/Latino } \\
\text { and other ethnicities }\end{array}$ & 87 & $8.7 \%$ & 96 & $9.6 \%$ & 93 & $9.3 \%$ & 92 & $9.2 \%$ & 88 & $8.8 \%$ & 86 & $8.6 \%$ & 542 & $9.0 \%$ \\
\hline White & 698 & $69.5 \%$ & 702 & $70.1 \%$ & 691 & $68.8 \%$ & 697 & $69.5 \%$ & 699 & $69.7 \%$ & 691 & $68.9 \%$ & 4179 & $69.4 \%$ \\
\hline Urban & 465 & $46.3 \%$ & 459 & $45.8 \%$ & 485 & $48.3 \%$ & 467 & $46.6 \%$ & 463 & $46.2 \%$ & 474 & $47.3 \%$ & 2812 & $46.7 \%$ \\
\hline Suburban & 382 & $38.0 \%$ & 379 & $37.8 \%$ & 369 & $36.7 \%$ & 365 & $36.4 \%$ & 376 & $37.5 \%$ & 365 & $36.4 \%$ & 2234 & $37.1 \%$ \\
\hline Rural & 158 & $15.7 \%$ & 164 & $16.4 \%$ & 151 & $15.0 \%$ & 171 & $17.0 \%$ & 164 & $16.4 \%$ & 164 & $16.4 \%$ & 969 & $16.1 \%$ \\
\hline Separated & 128 & $12.7 \%$ & 132 & $13.2 \%$ & 119 & $11.8 \%$ & 122 & $12.2 \%$ & 113 & $11.3 \%$ & 118 & $11.8 \%$ & 735 & $12.2 \%$ \\
\hline Married & 613 & $61.0 \%$ & 605 & $60.4 \%$ & 622 & $61.9 \%$ & 634 & $63.2 \%$ & 638 & $63.6 \%$ & 653 & $65.1 \%$ & 3763 & $62.5 \%$ \\
\hline Single & 251 & $25.0 \%$ & 251 & $25.0 \%$ & 253 & $25.2 \%$ & 233 & $23.2 \%$ & 239 & $23.8 \%$ & 216 & $21.5 \%$ & 1445 & $24.0 \%$ \\
\hline Age 18-39 & 394 & $39.2 \%$ & 389 & $38.8 \%$ & 394 & $39.2 \%$ & 388 & $38.7 \%$ & 390 & $38.9 \%$ & 392 & $39.1 \%$ & 2348 & $39.0 \%$ \\
\hline Age $40-59$ & 306 & $30.4 \%$ & 312 & $31.1 \%$ & 307 & $30.5 \%$ & 309 & $30.8 \%$ & 305 & $30.4 \%$ & 305 & $30.4 \%$ & 1842 & $30.6 \%$ \\
\hline Age 60+ & 305 & $30.3 \%$ & 301 & $30.0 \%$ & 304 & $30.2 \%$ & 306 & $30.5 \%$ & 308 & $30.7 \%$ & 306 & $30.5 \%$ & 1830 & $30.4 \%$ \\
\hline Total respondents & 1005 & $100 \%$ & 1002 & $100 \%$ & 1005 & $100 \%$ & 1003 & $100 \%$ & 1003 & $100 \%$ & 1003 & $100 \%$ & 6021 & $100 \%$ \\
\hline
\end{tabular}

Table 2 presents the models with two classes and their estimated membership proportions for each wave and for the full sample. The largest proportion of participants is found in Class 1 (75.6\%) with around $24.4 \%$ in Class 2. Each class corresponds to an underlying subgroup of participants that can be 
characterized by a particular pattern of mental health indicators during the COVID-19 pandemic. In particular, Class 2 appears to represent participants with high scores on all three mental health indicators (anxiety, feeling depressed, and feeling lonely). In this class, participants are more likely to be depressed, Ionely, and anxious, with probabilities around $0.8,0.7$, and 0.7 , respectively. As such, we will refer to Class 2 as the "high-symptoms class". In contrast, Class 1 contains participants with low scores on all three mental health indicators, who have a low probability of moderate to severe anxiety, feeling depressed, and feeling lonely (probabilities of $0.01,0.07$ and 0.06 , respectively). We refer to Class 1 as the "no/low-symptoms class." The characteristics of these two classes are consistent across the six waves. Table A.5 displays three classes' results: no/low-symptoms (68.9\% of participants), moderate-symptoms (14\%), and highsymptoms (17.1\%).

Sex-specific LCA analyses were conducted to determine whether the latent classes were different for men and women. The results reported in the two last panels of Table 2 show that patterns for men and women are similar to those found for the pooled sample, with a "no/low-symptoms" class and a "highsymptoms" class.

Table 2

Description of latent classes for the total sample, the women subsample, and the men subsample across waves

\begin{tabular}{|c|c|c|c|c|c|c|c|c|c|c|c|c|c|c|}
\hline & \multicolumn{2}{|c|}{ All waves } & \multicolumn{2}{|c|}{ Wave 1} & \multicolumn{2}{|c|}{ Wave 2} & \multicolumn{2}{|c|}{ Wave 3} & \multicolumn{2}{|c|}{ Wave 4} & \multicolumn{2}{|c|}{ Wave 5} & \multicolumn{2}{|c|}{ Wave 6} \\
\hline & $\begin{array}{l}\text { Class } \\
1\end{array}$ & $\begin{array}{l}\text { Class } \\
2\end{array}$ & $\begin{array}{l}\text { Class } \\
1\end{array}$ & $\begin{array}{l}\text { Class } \\
2\end{array}$ & $\begin{array}{l}\text { Class } \\
1\end{array}$ & $\begin{array}{l}\text { Class } \\
2\end{array}$ & $\begin{array}{l}\text { Class } \\
1\end{array}$ & $\begin{array}{l}\text { Class } \\
2\end{array}$ & $\begin{array}{l}\text { Class } \\
1\end{array}$ & $\begin{array}{l}\text { Class } \\
2\end{array}$ & $\begin{array}{l}\text { Class } \\
1\end{array}$ & $\begin{array}{l}\text { Class } \\
2\end{array}$ & $\begin{array}{l}\text { Class } \\
1\end{array}$ & $\begin{array}{l}\text { Class } \\
2\end{array}$ \\
\hline \multicolumn{15}{|l|}{ Total sample } \\
\hline $\begin{array}{l}\text { Proportion of respondents in } \\
\text { each class (\%) }\end{array}$ & 75.6 & 24.4 & 73.9 & 26.1 & 76.2 & 23.8 & 76.3 & 23.7 & 76.0 & 24.0 & 77.1 & 22.9 & 74.1 & 25.9 \\
\hline \multicolumn{15}{|l|}{ Proportion of respondents that } \\
\hline Felt depressed (\%) & 1.5 & 78.5 & 0.4 & 77.0 & 0.9 & 85.8 & 1.5 & 72.9 & 1.1 & 74.5 & 3.0 & 82.7 & 2.1 & 77.9 \\
\hline Felt lonely (\%) & 7.0 & 69.9 & 8.7 & 64.3 & 8.7 & 71.4 & 6.0 & 69.3 & 7.9 & 70.9 & 5.0 & 71.0 & 5.9 & 73.2 \\
\hline Felt anxious (\%) & 5.7 & 71.8 & 6.2 & 80.2 & 7.0 & 67.6 & 4.9 & 66.5 & 3.7 & 68.3 & 5.4 & 74.1 & 7.1 & 73.6 \\
\hline Number of respondents & 6,021 & & 1,005 & & 1,002 & & 1,005 & & 1,003 & & 1,003 & & 1,003 & \\
\hline \multicolumn{15}{|l|}{ Subsample of women } \\
\hline $\begin{array}{l}\text { Proportion of respondents in } \\
\text { each class }(\%)\end{array}$ & 73.0 & 27.0 & 70.6 & 29.4 & 76.1 & 23.9 & 73.9 & 26.1 & 72.0 & 28.0 & 70.8 & 29.2 & 72.7 & 27.3 \\
\hline \multicolumn{15}{|l|}{ Proportion of respondents that } \\
\hline Felt depressed (\%) & 2.3 & 78.7 & 1.8 & 79.0 & 2.9 & 87.3 & 3.8 & 75.0 & 0.0 & 71.9 & 1.4 & 77.7 & 3.6 & 79.3 \\
\hline Felt lonely (\%) & 8.2 & 69.7 & 9.7 & 69.5 & 9.7 & 75.0 & 6.8 & 69.5 & 9.8 & 60.6 & 6.0 & 65.2 & 6.8 & 77.2 \\
\hline Felt anxious (\%) & 6.4 & 71.6 & 8.1 & 80.8 & 7.3 & 74.3 & 5.6 & 64.6 & 3.2 & 63.6 & 5.2 & 70.5 & 8.6 & 72.4 \\
\hline Number of respondents & 2,987 & & 498 & & 497 & & 499 & & 492 & & 498 & & 503 & \\
\hline \multicolumn{15}{|l|}{ Subsample of men } \\
\hline $\begin{array}{l}\text { Proportion of respondents in } \\
\text { each class (\%) }\end{array}$ & 78.6 & 21.4 & 78.2 & 21.8 & 79.1 & 20.9 & 79.5 & 20.5 & 79.5 & 20.5 & 82.5 & 17.5 & 75.9 & 24.1 \\
\hline \multicolumn{15}{|l|}{ Proportion of respondents that } \\
\hline Felt depressed (\%) & 0.7 & 78.3 & 0.0 & 74.5 & 0.0 & 89.3 & 0.0 & 71.1 & 1.4 & 75.3 & 3.9 & 88.6 & 0.9 & 75.5 \\
\hline Felt lonely (\%) & 5.9 & 70.2 & 7.7 & 59.7 & 8.1 & 69.6 & 5.2 & 71.2 & 5.9 & 82.5 & 4.1 & 79.7 & 5.2 & 67.8 \\
\hline Felt anxious (\%) & 5.1 & 72.0 & 4.3 & 81.7 & 7.2 & 61.9 & 4.5 & 71.2 & 4.0 & 72.2 & 5.1 & 78.2 & 5.7 & 75.6 \\
\hline Number of respondents & 2,987 & & 504 & & 492 & & 501 & & 501 & & 497 & & 492 & \\
\hline
\end{tabular}

\section{Factors associated with high-symptoms class membership}

We used logistic regression to identify factors associated with class membership in the total sample and within each wave's data (see Table 3). We regressed the binary variable which indicated whether or not individuals were in the high-symptoms class based on individual and household characteristics. The adjusted odds ratios are reported in Table 3. From the total sample, women (aOR $=1.34,95 \% \mathrm{Cl}: 1.18-1.52)$ and participants with a post-secondary level of education ( $\mathrm{aOR}=1.26,95 \% \mathrm{Cl}: 1.02-1.55)$ were at greater odds of being in this class than men and those with a college diploma. Individuals who reported being worried about contracting COVID-19 were also at greater odds of being in the high symptoms class (aOR $=2.39,95 \% \mathrm{Cl}$ : 2.02-2.82). People who were married $(\mathrm{aOR}=0.71,95 \% \mathrm{Cl}: 0.59-0.85)$, aged $60+(\mathrm{aOR}=0.38,95 \% \mathrm{Cl}: 0.32-0.47)$ and with incomes higher than CAD $\$ 40,000$ : between $\$ 40,000-\$ 79,000(\mathrm{aOR}=$ $0.73,95 \% \mathrm{Cl}: 0.60-0.90), \$ 80,000-\$ 119,000(\mathrm{aOR}=0.60,95 \% \mathrm{Cl}: 0.48-0.74)$ and $\$ 120,000+(\mathrm{aOR}=0.47,95 \% \mathrm{Cl}: 0.37-0.59)$ were less likely than single, younger age groups, and people in lower income groups, to be in the high-symptoms class. Those who self-identified as Asian (aOR $=0.78,95 \% \mathrm{Cl}$ : $0.62-0.97)$ were less likely to be in the high-symptoms class compared to people who self-identified as Black/Indigenous/other ethnicities. 
Across waves, the results show some heterogeneity suggesting that risk factors for reporting a high-symptoms level of mental health may vary at different time points. For example, women's odds ratios were significantly greater than one in Waves 1,3 , or $5(\mathrm{aOR}=1.56,95 \% \mathrm{Cl}: 1.14-2.12$; $\mathrm{aOR}=1.48,95 \% \mathrm{Cl}$ : $1.08-$ $2.03 ; \mathrm{aOR}=1.63,95 \% \mathrm{Cl}: 1.14-2.31$ ) and not significantly different from zero in Waves 2,4 , and 6 . Note that individuals who worried about contracting COVID19 were consistently at greater odds of being in the high-symptoms class, whereas those aged $60+$ were consistently less likely to be in this group. 
Table 3

Factors associated with high-level mental health symptoms class membership (Adjusted Odds Ratios)

\begin{tabular}{|c|c|c|c|c|c|c|c|}
\hline & Wave 1 & Wave 2 & Wave 3 & Wave 4 & Wave5 & Wave 6 & all waves \\
\hline \multirow[t]{2}{*}{ Women } & $1.56^{\star \star \star}$ & 1.22 & $1.48^{\star *}$ & 1.17 & $1.63^{\star * *}$ & 1.15 & $1.34^{\star \star \star}$ \\
\hline & $\begin{array}{l}(1.14- \\
2.12)\end{array}$ & $\begin{array}{l}(0.88- \\
1.67)\end{array}$ & $\begin{array}{l}(1.08- \\
2.03)\end{array}$ & $\begin{array}{l}(0.86- \\
1.61)\end{array}$ & $\begin{array}{l}(1.14- \\
2.31)\end{array}$ & $\begin{array}{l}(0.85- \\
1.56)\end{array}$ & $\begin{array}{l}(1.18- \\
1.52)\end{array}$ \\
\hline \multirow[t]{2}{*}{ Worry about contracting COVID-19 } & $3.61^{\star \star \star}$ & $2.40 * \star \star$ & $1.73^{\star * *}$ & $2.50 * \star \star$ & $3.10 * \star \star$ & $2.01^{\star \star \star}$ & $2.39 * \star \star$ \\
\hline & $\begin{array}{l}(2.27- \\
5.75)\end{array}$ & $\begin{array}{l}(1.62- \\
3.57)\end{array}$ & $\begin{array}{l}(1.18- \\
2.53)\end{array}$ & $\begin{array}{l}(1.66- \\
3.75)\end{array}$ & $\begin{array}{l}(1.88- \\
5.09)\end{array}$ & $\begin{array}{l}(1.33- \\
3.05)\end{array}$ & $\begin{array}{l}(2.02- \\
2.82)\end{array}$ \\
\hline \multirow[t]{2}{*}{ Living with others } & 1.07 & 0.95 & 1.54 & 0.82 & 0.74 & 1.18 & 1.03 \\
\hline & $\begin{array}{l}(0.65- \\
1.77)\end{array}$ & $\begin{array}{l}(0.57- \\
1.57)\end{array}$ & $\begin{array}{l}(0.95- \\
2.52)\end{array}$ & $\begin{array}{l}(0.50- \\
1.35)\end{array}$ & $\begin{array}{l}(0.44- \\
1.24)\end{array}$ & $\begin{array}{l}(0.72- \\
1.92)\end{array}$ & $\begin{array}{l}(0.85- \\
1.26)\end{array}$ \\
\hline \multirow[t]{2}{*}{ Presence of children } & 1.14 & 1.17 & 0.73 & 1.27 & $1.96^{\star \star \star}$ & 0.87 & 1.14 \\
\hline & $\begin{array}{l}(0.77- \\
1.69)\end{array}$ & $\begin{array}{l}(0.80- \\
1.73)\end{array}$ & $\begin{array}{l}(0.47- \\
1.12)\end{array}$ & $\begin{array}{l}(0.85- \\
1.91)\end{array}$ & $\begin{array}{l}(1.26- \\
3.05)\end{array}$ & $\begin{array}{l}(0.58- \\
1.31)\end{array}$ & $\begin{array}{l}(0.97- \\
1.35)\end{array}$ \\
\hline Income $\leq \$ 40,000$ & REF & REF & REF & REF & REF & REF & REF \\
\hline \multirow[t]{2}{*}{ Income of $\$ 40,000-\$ 79,999$} & 0.74 & 0.73 & 0.68 & 0.98 & $0.55^{\star \star}$ & 0.67 & $0.73^{\star \star \star}$ \\
\hline & $\begin{array}{l}(0.44- \\
1.22)\end{array}$ & $\begin{array}{l}(0.44- \\
1.19)\end{array}$ & $\begin{array}{l}(0.42- \\
1.12)\end{array}$ & $\begin{array}{l}(0.59- \\
1.63)\end{array}$ & $\begin{array}{l}(0.31- \\
0.98)\end{array}$ & $\begin{array}{l}(0.40- \\
1.12)\end{array}$ & $\begin{array}{l}(0.60- \\
0.90)\end{array}$ \\
\hline \multirow[t]{2}{*}{ Income of $\$ 80,000-\$ 119,999$} & 0.86 & $0.40 * \star \star$ & $0.52^{\star *}$ & 0.73 & $0.42^{\star * *}$ & 0.58 & 0.60 *** \\
\hline & $\begin{array}{l}(0.51- \\
1.48)\end{array}$ & $\begin{array}{l}(0.23- \\
0.71)\end{array}$ & $\begin{array}{l}(0.30- \\
0.89)\end{array}$ & $\begin{array}{l}(0.42- \\
1.28)\end{array}$ & $\begin{array}{l}(0.23- \\
0.76)\end{array}$ & $\begin{array}{l}(0.34- \\
1.01)\end{array}$ & $\begin{array}{l}(0.48- \\
0.74)\end{array}$ \\
\hline \multirow[t]{2}{*}{ Income $\$ 120,000+$} & 0.63 & $0.23^{\star \star \star}$ & $0.29 * \star \star$ & 0.87 & 0.54 & 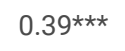 & $0.47^{\star \star \star}$ \\
\hline & $\begin{array}{l}(0.35- \\
1.11)\end{array}$ & $\begin{array}{l}(0.13- \\
0.41)\end{array}$ & $\begin{array}{l}(0.16- \\
0.53)\end{array}$ & $\begin{array}{l}(0.50- \\
1.53)\end{array}$ & $\begin{array}{l}(0.30- \\
1.00)\end{array}$ & $\begin{array}{l}(0.22- \\
0.69)\end{array}$ & $\begin{array}{l}(0.37- \\
0.59)\end{array}$ \\
\hline \multirow[t]{2}{*}{ Income missing } & 0.61 & 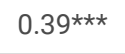 & $0.45^{\star \star \star}$ & 0.73 & $0.41^{\star \star \star}$ & 0.58 & $0.55^{\star \star \star}$ \\
\hline & $\begin{array}{l}(0.34- \\
1.10)\end{array}$ & $\begin{array}{l}(0.21- \\
0.72)\end{array}$ & $\begin{array}{l}(0.25- \\
0.82)\end{array}$ & $\begin{array}{l}(0.41- \\
1.28)\end{array}$ & $\begin{array}{l}(0.22- \\
0.77)\end{array}$ & $\begin{array}{l}(0.33- \\
1.04)\end{array}$ & $\begin{array}{l}(0.44- \\
0.69)\end{array}$ \\
\hline College diploma & REF & REF & REF & REF & REF & REF & REF \\
\hline \multirow[t]{2}{*}{ High school } & 1.11 & 0.73 & 0.85 & 1.04 & 1.82 & 0.83 & 0.99 \\
\hline & $\begin{array}{l}(0.62- \\
1.96)\end{array}$ & $\begin{array}{l}(0.40- \\
1.36)\end{array}$ & $\begin{array}{l}(0.48- \\
1.50)\end{array}$ & $\begin{array}{l}(0.58- \\
1.85)\end{array}$ & $\begin{array}{l}(0.96- \\
3.44)\end{array}$ & $\begin{array}{l}(0.46- \\
1.48)\end{array}$ & $\begin{array}{l}(0.79- \\
1.26)\end{array}$ \\
\hline \multirow[t]{2}{*}{ Post-secondary } & 1.11 & 1.42 & 1.09 & 0.93 & 1.96 ** & 1.22 & $1.26^{\star \star}$ \\
\hline & $\begin{array}{l}(0.67- \\
1.84)\end{array}$ & $\begin{array}{l}(0.86- \\
2.37)\end{array}$ & $\begin{array}{l}(0.64- \\
1.85)\end{array}$ & $\begin{array}{l}(0.55- \\
1.58)\end{array}$ & $\begin{array}{l}(1.07- \\
3.59)\end{array}$ & $\begin{array}{l}(0.74- \\
2.00)\end{array}$ & $\begin{array}{l}(1.02- \\
1.55)\end{array}$ \\
\hline \multirow[t]{2}{*}{ University } & 0.79 & 0.98 & 0.95 & 1.09 & 1.13 & 1.19 & 1.01 \\
\hline & $\begin{array}{l}(0.53- \\
1.19)\end{array}$ & $\begin{array}{l}(0.64- \\
1.48)\end{array}$ & $\begin{array}{l}(0.61- \\
1.47)\end{array}$ & $\begin{array}{l}(0.72- \\
1.63)\end{array}$ & $\begin{array}{l}(0.70- \\
1.81)\end{array}$ & $\begin{array}{l}(0.80- \\
1.77)\end{array}$ & $\begin{array}{l}(0.85- \\
1.19)\end{array}$ \\
\hline $\begin{array}{l}\text { Black/Indigenous/Arab/Latino and other } \\
\text { ethnicities }\end{array}$ & REF & REF & REF & REF & REF & REF & REF \\
\hline \multirow[t]{2}{*}{ Asian } & 0.64 & 1.13 & $0.52^{\star *}$ & 0.91 & 0.93 & 0.65 & $0.78^{\star \star}$ \\
\hline & $\begin{array}{l}(0.37- \\
1.12)\end{array}$ & $\begin{array}{l}(0.64- \\
2.02)\end{array}$ & $\begin{array}{l}(0.30- \\
0.90)\end{array}$ & $\begin{array}{l}(0.52- \\
1.58)\end{array}$ & $\begin{array}{l}(0.50- \\
1.72)\end{array}$ & $\begin{array}{l}(0.38- \\
1.11)\end{array}$ & $\begin{array}{l}(0.62- \\
0.97)\end{array}$ \\
\hline \multirow[t]{2}{*}{ White } & 0.70 & 1.24 & $0.58^{\star *}$ & 0.80 & 0.92 & 0.84 & 0.83 \\
\hline & $\begin{array}{l}(0.44- \\
1.14)\end{array}$ & $\begin{array}{l}(0.75- \\
2.05)\end{array}$ & $\begin{array}{l}(0.36- \\
0.93)\end{array}$ & $\begin{array}{l}(0.49- \\
1.31)\end{array}$ & $\begin{array}{l}(0.53- \\
1.60)\end{array}$ & $\begin{array}{l}(0.53- \\
1.34)\end{array}$ & $\begin{array}{l}(0.69- \\
1.01)\end{array}$ \\
\hline Rural & REF & REF & REF & REF & REF & REF & REF \\
\hline \multirow[t]{2}{*}{ Urban } & 1.47 & 1.05 & 1.23 & 0.77 & 1.68 & 0.73 & 1.13 \\
\hline & $\begin{array}{l}(0.91- \\
2.37)\end{array}$ & $\begin{array}{l}(0.65- \\
1.70)\end{array}$ & $\begin{array}{l}(0.75- \\
2.04)\end{array}$ & $\begin{array}{l}(0.49- \\
1.21)\end{array}$ & $\begin{array}{l}(0.99- \\
2.85)\end{array}$ & $\begin{array}{l}(0.47- \\
1.14)\end{array}$ & $\begin{array}{l}(0.93- \\
1.36)\end{array}$ \\
\hline Suburban & 1.25 & 1.01 & 0.95 & 0.76 & 0.94 & 0.85 & 0.98 \\
\hline
\end{tabular}




\begin{tabular}{|c|c|c|c|c|c|c|c|}
\hline & Wave 1 & Wave 2 & Wave 3 & Wave 4 & Wave5 & Wave 6 & all waves \\
\hline & $\begin{array}{l}(0.76- \\
2.06)\end{array}$ & $\begin{array}{l}(0.62- \\
1.67)\end{array}$ & $\begin{array}{l}(0.57- \\
1.60)\end{array}$ & $\begin{array}{l}(0.48- \\
1.22)\end{array}$ & $\begin{array}{l}(0.54- \\
1.64)\end{array}$ & $\begin{array}{l}(0.55- \\
1.33)\end{array}$ & $\begin{array}{l}(0.80- \\
1.19)\end{array}$ \\
\hline Single & REF & REF & REF & REF & REF & REF & REF \\
\hline \multirow[t]{2}{*}{ Separated } & 1.28 & 1.53 & $1.87 * *$ & 0.90 & 0.65 & 1.17 & 1.14 \\
\hline & $\begin{array}{l}(0.72- \\
2.27)\end{array}$ & $\begin{array}{l}(0.87- \\
2.68)\end{array}$ & $\begin{array}{l}(1.04- \\
3.36)\end{array}$ & $\begin{array}{l}(0.52- \\
1.57)\end{array}$ & $\begin{array}{l}(0.33- \\
1.28)\end{array}$ & $\begin{array}{l}(0.66- \\
2.06)\end{array}$ & $\begin{array}{l}(0.90- \\
1.43)\end{array}$ \\
\hline \multirow[t]{2}{*}{ Married } & 0.89 & 1.06 & 0.75 & $0.55^{\star \star}$ & $0.47^{* * *}$ & 0.77 & $0.71^{\star \star \star}$ \\
\hline & $\begin{array}{l}(0.57- \\
1.40)\end{array}$ & $\begin{array}{l}(0.68- \\
1.65)\end{array}$ & $\begin{array}{l}(0.46- \\
1.20)\end{array}$ & $\begin{array}{l}(0.34- \\
0.87)\end{array}$ & $\begin{array}{l}(0.29- \\
0.76)\end{array}$ & $\begin{array}{l}(0.48- \\
1.22)\end{array}$ & $\begin{array}{l}(0.59- \\
0.85)\end{array}$ \\
\hline Age 18-39 & REF & REF & REF & REF & REF & REF & REF \\
\hline \multirow[t]{2}{*}{ Age $40-59$} & 0.79 & 0.92 & 1.00 & 0.75 & 0.96 & 0.92 & 0.90 \\
\hline & $\begin{array}{l}(0.55- \\
1.13)\end{array}$ & $\begin{array}{l}(0.64- \\
1.33)\end{array}$ & $\begin{array}{l}(0.68- \\
1.47)\end{array}$ & $\begin{array}{l}(0.51- \\
1.09)\end{array}$ & $\begin{array}{l}(0.65- \\
1.42)\end{array}$ & $\begin{array}{l}(0.64- \\
1.32)\end{array}$ & $\begin{array}{l}(0.78- \\
1.05)\end{array}$ \\
\hline \multirow[t]{2}{*}{ Age 60+ } & $0.38^{\star \star \star}$ & $0.26^{\star \star \star}$ & $0.36^{\star \star \star}$ & $0.47^{\star \star \star}$ & $0.27^{\star \star \star}$ & $0.34 * \star \star$ & $0.38^{\star \star \star}$ \\
\hline & $\begin{array}{l}(0.24- \\
0.61)\end{array}$ & $\begin{array}{l}(0.15- \\
0.44)\end{array}$ & $\begin{array}{l}(0.22- \\
0.60)\end{array}$ & $\begin{array}{l}(0.29- \\
0.77)\end{array}$ & $\begin{array}{l}(0.15- \\
0.51)\end{array}$ & $\begin{array}{l}(0.22- \\
0.55)\end{array}$ & $\begin{array}{l}(0.32- \\
0.47)\end{array}$ \\
\hline \multirow[t]{2}{*}{ Wave 1} & & & & & & & 0.92 \\
\hline & & & & & & & $\begin{array}{l}(0.74- \\
1.14)\end{array}$ \\
\hline \multirow[t]{2}{*}{ Wave 2} & & & & & & & 0.93 \\
\hline & & & & & & & $\begin{array}{l}(0.75- \\
1.15)\end{array}$ \\
\hline \multirow[t]{2}{*}{ Wave 3} & & & & & & & 0.83 \\
\hline & & & & & & & $\begin{array}{l}(0.67- \\
1.03)\end{array}$ \\
\hline \multirow[t]{2}{*}{ Wave 4} & & & & & & & 0.86 \\
\hline & & & & & & & $\begin{array}{l}(0.69- \\
1.07)\end{array}$ \\
\hline \multirow[t]{2}{*}{ Wave 5} & & & & & & & 0.91 \\
\hline & & & & & & & $\begin{array}{l}(0.74- \\
1.13)\end{array}$ \\
\hline Wave 6 & REF & REF & REF & REF & REF & REF & REF \\
\hline \multirow[t]{2}{*}{ Constant } & $0.18^{\star \star \star}$ & $0.31^{\star \star \star}$ & 0.47 & 0.44 & $0.21^{\star \star \star}$ & 0.57 & $0.37^{\star \star \star}$ \\
\hline & $\begin{array}{l}(0.07- \\
0.48)\end{array}$ & $\begin{array}{l}(0.13- \\
0.75)\end{array}$ & $\begin{array}{l}(0.19- \\
1.13)\end{array}$ & $\begin{array}{l}(0.18- \\
1.09)\end{array}$ & $\begin{array}{l}(0.08- \\
0.59)\end{array}$ & $\begin{array}{l}(0.24- \\
1.37)\end{array}$ & $\begin{array}{l}(0.25- \\
0.55)\end{array}$ \\
\hline Observations & 1,002 & 999 & 1,000 & 1,000 & 1,001 & 997 & 5,999 \\
\hline Pseudo R-squared & 0.077 & 0.097 & 0.091 & 0.066 & 0.148 & 0.058 & 0.069 \\
\hline
\end{tabular}

\section{Associations of high-symptoms class membership with alcohol and cannabis use}

Table 4 displays associations of class membership with alcohol and cannabis use in the total sample and by sex, controlling for sex, age, marital status, education, ethnicity, living area, household confounders, worrying about getting COVID-19, and survey wave indicator variables. Individuals in the highsymptoms class were at greater odds of using cannabis at least once a week and frequently engaging in heavy episodic drinking (aOR $=2.25,95 \% \mathrm{Cl}: 1.90-$ $2.67 ; \mathrm{aOR}=1.69,95 \% \mathrm{Cl}: 1.47-1.95)$ relative to those in the no/low-symptoms class. Regarding changes in cannabis and alcohol consumption, results indicated that being in the high-symptoms class was associated with greater odds of increasing cannabis and alcohol use during the pandemic (aOR $=3.48$, 95\%Cl:2.79-4.35; aOR = 2.37, 95\%Cl:2.05-2.73). 
Table 4

Associations of high-symptoms class membership with alcohol and cannabis use during the pandemic (Adjusted odds ratios)

\begin{tabular}{|c|c|c|c|c|}
\hline & Cannabis use past week & Heavy episodic drinking & Increase in cannabis use & Increase in alcohol use \\
\hline \multicolumn{5}{|l|}{ Total sample } \\
\hline \multirow[t]{2}{*}{ High-symptoms class } & $2.25^{\star \star \star}$ & $1.69 \star \star \star$ & $3.48^{\star \star \star}$ & $2.37 * \star \star$ \\
\hline & $(1.90-2.67)$ & $(1.47-1.95)$ & $(2.79-4.35)$ & $(2.05-2.73)$ \\
\hline \multicolumn{5}{|l|}{ Subsample of women } \\
\hline \multirow[t]{2}{*}{ High-symptoms class } & $1.96 * \star \star$ & $1.66^{\star \star \star}$ & $2.87^{\star \star \star}$ & $2.09 \star \star \star$ \\
\hline & $(1.53-2.52)$ & $(1.37-2.02)$ & $(2.04-4.03)$ & $(1.71-2.56)$ \\
\hline \multicolumn{5}{|l|}{ Subsample of men } \\
\hline \multirow[t]{2}{*}{ High-symptoms class } & $2.53^{\star \star \star}$ & $1.72^{\star \star \star}$ & $3.87 \star \star \star$ & $2.62^{\star \star \star}$ \\
\hline & $(2.00-3.20)$ & $(1.40-2.10)$ & $(2.86-5.25)$ & $(2.12-3.24)$ \\
\hline \multicolumn{5}{|c|}{ Legend: $95 \%$ confidence level in parentheses. Significance level $* \star * p<0.01, * \star p<0.05$. } \\
\hline
\end{tabular}

Table 5 reported aORs controlling for high-symptoms class*women interactions to test if high-symptoms class interacts with sex. Results show that aORs were similar for men and women, except for increase in alcohol use - suggesting that women with high-symptoms were at greater odds of increasing alcohol drinking during the pandemic than men with high-symptoms.

Table 5

Adjusted odds ratios from model with sex and latent class membership interaction

\begin{tabular}{|c|c|c|c|c|}
\hline & Cannabis use past week & Heavy Episodic Drinking & Increase in cannabis use & Increase in alcohol use \\
\hline \multirow[t]{2}{*}{ High-symptoms class } & $2.52^{\star \star \star}$ & $1.75^{\star \star \star}$ & $4.01^{\star \star \star}$ & $2.82^{\star \star \star}$ \\
\hline & $(2.00-3.17)$ & $(1.44-2.13)$ & $(3.00-5.37)$ & $(2.30-3.47)$ \\
\hline \multirow[t]{2}{*}{ High-symptoms classwomen } & 0.79 & 0.94 & 0.72 & $0.72^{\star \star}$ \\
\hline & $(0.57-1.10)$ & $(0.72-1.22)$ & $(0.46-1.11)$ & $(0.54-0.95)$ \\
\hline \multicolumn{5}{|c|}{ Legend: $95 \%$ confidence level in parentheses. Significance level $* \star \star p<0.01, * \star p<0.05$. } \\
\hline
\end{tabular}

Table A.6 displays similar results when considering three classes although the magnitude of the associations are smaller. Note that using anxiety, depression and loneliness variables as dependent variables to estimate their associations with alcohol and cannabis use did not provide consistent evidence particularly by sex (see Table A.7 in the Appendix).

Finally, we investigated whether the associations between class membership and cannabis and alcohol consumption varied across the survey waves. The results are in Table 6. The t-test for each interaction term (high-symptoms class*wave) indicates that the association between class membership and cannabis use/heavy episodic drinking (at the level of 5\%) do not change across waves. Similar results are observed for increase in cannabis use. For increase in alcohol use, two interaction terms: high-symptoms class*wave1 and high-symptoms class*wave 5 were statistically different from zero, suggesting that the odds ratios were significantly greater for Wave 1 and Wave 5 than for the other waves. To test if the odds ratios across waves are all statistically equal, we tested the null hypothesis that all the coefficients of the interaction terms are zero, using an F-test separating the total sample, and the man subsample, and the woman subsample. All the F-tests results (Table 6) have p-values greater than $5 \%$, except for increase in alcohol use ( $p$-value $<0.05$ in the total sample and man subsample). This suggests that, overall, being in the high-symptoms class for alcohol and cannabis use was not affected by time. However, we rejected that all the interaction terms were simultaneously zero for increase in alcohol use for the total sample and the man subsample, suggesting that the association of high-symptoms class membership and the change in drinking behaviour varies across time, particularly for men. 
Table 6

Adjusted odds ratios from model with time and latent class membership interaction

\begin{tabular}{|c|c|c|c|c|}
\hline & Cannabis use past week & Heavy Episodic Drinking & Increase in cannabis use & Increase in alcohol use \\
\hline \multicolumn{5}{|l|}{ Total sample } \\
\hline \multirow[t]{2}{*}{ High-symptoms class } & 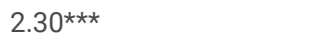 & $1.99 \star \star \star$ & $3.84 * \star \star$ & $3.38 * \star \star$ \\
\hline & $(1.59-3.33)$ & $(1.44-2.73)$ & $(2.42-6.10)$ & $(2.41-4.73)$ \\
\hline \multirow[t]{2}{*}{ High-symptoms classwave 1} & 0.95 & 1.13 & 0.60 & $0.61^{\star \star}$ \\
\hline & $(0.54-1.64)$ & $(0.72-1.77)$ & $(0.30-1.21)$ & $(0.38-0.97)$ \\
\hline \multirow[t]{2}{*}{ High-symptoms class*wave 2} & 0.84 & 0.77 & 1.04 & 0.79 \\
\hline & $(0.49-1.46)$ & $(0.49-1.21)$ & $(0.52-2.08)$ & $(0.49-1.26)$ \\
\hline \multirow[t]{2}{*}{ High-symptoms classwave 3} & 0.91 & 0.77 & 0.74 & 0.65 \\
\hline & $(0.52-1.58)$ & $(0.48-1.21)$ & $(0.37-1.52)$ & $(0.40-1.06)$ \\
\hline \multirow[t]{2}{*}{ High-symptoms class*wave 4} & 0.97 & 0.84 & 0.90 & 0.78 \\
\hline & $(0.56-1.67)$ & $(0.53-1.33)$ & $(0.45-1.83)$ & $(0.48-1.26)$ \\
\hline \multirow[t]{2}{*}{ High-symptoms class*wave 5} & 1.26 & 0.67 & 1.30 & $0.44^{\star \star \star}$ \\
\hline & $(0.72-2.19)$ & $(0.43-1.06)$ & $(0.61-2.77)$ & $(0.27-0.73)$ \\
\hline F-test chi2 statistics & 2.15 & 6.68 & 4.66 & 12.11 \\
\hline F-test p_value & 0.828 & 0.246 & 0.458 & 0.033 \\
\hline \multicolumn{5}{|l|}{ Subsample of women } \\
\hline \multirow[t]{2}{*}{ High-symptoms class } & $1.98^{\star \star}$ & $1.67 \star \star$ & $3.12^{\star \star \star}$ & $2.31 * \star \star$ \\
\hline & $(1.11-3.52)$ & $(1.06-2.62)$ & $(1.53-6.35)$ & $(1.44-3.68)$ \\
\hline \multirow[t]{2}{*}{ High-symptoms class*wave 1} & 0.99 & 1.35 & 0.42 & 0.78 \\
\hline & $(0.42-2.30)$ & $(0.71-2.57)$ & $(0.15-1.19)$ & $(0.41-1.47)$ \\
\hline \multirow[t]{2}{*}{ High-symptoms class*wave 2} & 0.93 & 0.98 & 1.18 & 0.77 \\
\hline & $(0.41-2.09)$ & $(0.50-1.93)$ & $(0.41-3.42)$ & $(0.39-1.50)$ \\
\hline \multirow[t]{2}{*}{ High-symptoms class*wave 3} & 0.71 & 0.96 & 0.68 & 1.14 \\
\hline & $(0.31-1.62)$ & $(0.51-1.80)$ & $(0.23-2.02)$ & $(0.59-2.20)$ \\
\hline \multirow[t]{2}{*}{ High-symptoms classwave 4} & 1.02 & 0.97 & 1.09 & 1.23 \\
\hline & $(0.43-2.44)$ & $(0.51-1.87)$ & $(0.33-3.65)$ & $(0.62-2.44)$ \\
\hline \multirow[t]{2}{*}{ High-symptoms classwave 5} & 1.47 & 0.80 & 1.83 & 0.67 \\
\hline & $(0.64-3.37)$ & $(0.41-1.53)$ & $(0.56-5.96)$ & $(0.33-1.34)$ \\
\hline F-test chi2 statistics & 2.90 & 2.64 & 6.96 & 4.76 \\
\hline F-test p_value & 0.715 & 0.756 & 0.224 & 0.446 \\
\hline \multicolumn{5}{|l|}{ Subsample of men } \\
\hline \multirow[t]{2}{*}{ High-symptoms class } & $2.47^{\star \star \star}$ & $2.24^{\star \star \star}$ & 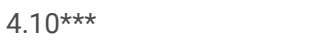 & $4.74^{\star \star \star}$ \\
\hline & $(1.49-4.09)$ & $(1.41-3.56)$ & $(2.19-7.68)$ & $(2.87-7.83)$ \\
\hline \multirow[t]{2}{*}{ High-symptoms class*wave 1} & 1.02 & 1.06 & 0.84 & 0.52 \\
\hline & $(0.48-2.17)$ & $(0.55-2.05)$ & $(0.33-2.16)$ & $(0.26-1.04)$ \\
\hline \multirow[t]{2}{*}{ High-symptoms class*wave 2} & 0.70 & 0.64 & 0.94 & 0.80 \\
\hline & $(0.32-1.55)$ & $(0.33-1.23)$ & $(0.36-2.44)$ & $(0.40-1.58)$ \\
\hline \multirow[t]{2}{*}{ High-symptoms class*wave 3} & 1.19 & 0.59 & 0.88 & $0.36^{\star \star *}$ \\
\hline & $(0.55-2.60)$ & $(0.30-1.16)$ & $(0.34-2.33)$ & $(0.17-0.74)$ \\
\hline \multirow[t]{2}{*}{ High-symptoms class*wave 4} & 1.08 & 0.75 & 0.93 & 0.52 \\
\hline & $(0.52-2.25)$ & $(0.39-1.47)$ & $(0.37-2.32)$ & $(0.26-1.05)$ \\
\hline
\end{tabular}




\begin{tabular}{|llll|}
\hline & Cannabis use past week & Heavy Episodic Drinking & Increase in cannabis use \\
\hline High-symptoms class*wave 5 & 1.29 & 0.64 & 1.09 \\
\hline & $(0.60-2.78)$ & $(0.33-1.24)$ & $(0.40-2.99)$ \\
\hline F-test chi2 statistics & 2.37 & 5.41 & 0.29 \\
\hline F-test p_value & 0.796 & 0.368 & 0.998 \\
\hline $\begin{array}{l}\text { Legend: } 95 \% \text { confidence level in parentheses. Significance level *** } p<0.01, * * \\
\text { Note: } \text { Odds ratios are adjusted for sex, age, marital status, education, ethnicity, living area, household income, the presence of children, and other people in } \\
\text { the household (see the Appendix for the full estimation table and the model specification). }\end{array}$ \\
\hline
\end{tabular}

\section{Discussion}

We found two classes of individuals: those with high scores on all three mental health indicators and those with no/low symptoms. The two classes were consistently identified across survey waves, which suggested that the classification was robust. The high-symptoms class was our class of interest and it contained around $23-26 \%$ of the participants with a high probability of being anxious, feeling depressed, and feeling lonely.

We found that women and participants with a post-secondary education level were more likely to be in the high-symptoms class, while Asians, married, senior individuals, and participants in households with incomes higher than CAD $\$ 40,000$ were less likely to belong to the high-symptoms class. Individuals worried about getting COVID-19 were also more likely to belong to the high-symptoms class. Additionally, we showed that high-symptoms class membership was associated with increased odds of using cannabis and heavy episodic drinking relative to the no/low-symptoms class. Increases in cannabis as well as alcohol use were also associated with class membership. These effects did not differ across survey waves.

These findings should be considered in the context of several limitations. First, although quota sampling is the non-probability sampling method that is the closest in representativeness to probability sampling, (49) its non-randomness may lead to potential selection bias (50). However, comparing quota and probability sampling, Cumming (1990) (51) found that quota sampling with age and sex quota controls may be an acceptable alternative to probability sampling. Second, our results may not be generalizable to the general population. Finally, cross-sectional data were collected; therefore, conclusions regarding causal relationships could not be made. Nevertheless, the study offers useful insights into understanding mental health and substance use co-morbidities and multi-morbidities during the COVID-19 pandemic.

Our first finding clearly identifies a group of individuals who experienced high-level mental health symptoms, and suggests that the well-established comorbidity of anxiety and depression might also coexist with feelings of loneliness during the COVID-19 pandemic. This finding is consistent with previous studies demonstrating an association between loneliness, depression, anxiety and their co-morbidity $(13,14,52)$. The second main finding reveals that worrying about contracting COVID-19 (and/or fear of someone close getting COVID-19) was the main risk factor for experiencing high-level mental health symptoms. This result suggests that mental health symptoms during the pandemic could be reduced by reducing the fear of COVID-19 within the population. Effective communication strategies employed during the pandemic from governments or public health authorities might help to enhance the long-term psychological well-being of people and mitigate the fear of contracting the COVID-19 virus (53-55).

The fourth main finding reveals that people (regardless of their sex) who are at a high-symptoms level (compared to no/low-symptoms) were more likely to increase the use of cannabis and alcohol during the pandemic, suggesting that people with a high-symptoms level may be turning to substances to help alleviate negative symptoms. Women tend to increase their alcohol use during the pandemic that men. This finding corroborates the fact that COVID-19 psychological distress is associated to drinking more in women than men (56). However, using alcohol and cannabis to deal with symptoms of anxiety and depression or with life challenges can increase the risk of developing alcohol or cannabis use disorder, or both (57). Moreover, in the longer term, substance misuse can worsen these emotional disorder symptoms. This implies that treatment programs are needed to better address the co-morbid disorders in response to the mental health effects of the pandemic.

Our results suggest that initiatives (e.g., screening, virtual consultation) aimed at improving population mental health and substance use problems during the pandemic should be adapted to account for sex, age, ethnicity, marital status, and socioeconomic status, while prioritizing women and post-secondaryeducated individuals. These initiatives should also integrate effective communication strategies with the goal of reducing people's fear of contracting the virus, and encouraging behaviors that reduce the spread of COVID-19. The results also suggest that each psychiatric consultation should be paired with screening for substance use disorders, particularly women should be screen for alcohol misuse.

\section{Conclusions}

We identified an important group of individuals with high levels of anxiety, depression, and loneliness during the COVID-19 pandemic who tended to drink more alcohol and use more cannabis compared to those with no/low-symptoms. This finding suggests that initiatives and supports are needed to address mental health and substance use multi-morbidities, particularly during the COVID-19 pandemic.

\section{Abbreviations}

COVID-19

Coronavirus disease 2019 
LCA

Latent class analysis

GAD

generalized anxiety disorder

CES-D

Center for Epidemiologic Studies Depression

AIC

Akaike information criterion

BIC

Bayesian information criterion

$\mathrm{aOR}$

Adjusted odds ratio

$\mathrm{Cl}$

Confidence interval

RR

Response rate

\section{Declarations}

\section{Ethics approval and consent to participate}

The study has been granted ethics committee approval from the Research Ethics Board at the Centre for Addiction and Mental Health, Toronto, ON, Canada. The Centre For Addiction and Mental Health Research Ethics Board (CAMH REB) operates in compliance with, and is constituted in accordance with, the requirements of the Tri-Council Policy Statement: Ethical Conduct for Research Involving Humans (TCPS 2), the International Conference on Harmonisation Good Clinical Practice Consolidated Guideline (ICH GCP), Part C, Division 5 of the Food and Drug Regulations, Part 4 of the Natural Health Products Regulations, Part 3 of the Medical Devices Regulations, and the provisions of the Ontario Personal Health Information Protection Act (PHIPA 2004) and its applicable regulations. The CAMH REB is qualified through the CTO REB Qualification Program and is registered with the U.S. Department of Health and Human Services (DHHS) Office for Human Research Protection (OHRP). All participants provided a written consent to participate.

\section{Consent for publication}

N.A.

\section{Availability of data}

All data generated or analysed during this study are included in this published article [and its supplementary information files]. Data are also publicly available for download at: http://www.delvinia.com/coronavirus/.

\section{Competing interests}

The authors declared no potential conflicts of interest with respect to the research, authorship, and/or publication of this article.

\section{Funding}

Delvinia, a research technology firm provided in-kind support for data collection. We did not receive funding from Delvinia, however, they have administered our questionnaires to Canadians through their web-based panel AskingCanadians (http://www.delvinia.com/solutions/askingcanadians/), without charging any fees.

\section{Authors' contributions}

JR, SA, SW and NHS came up the paper idea. SW, DF, SA, and NHS conceptualized and designed the study. HAH and TEM developed the survey questionnaires for the data collection. NHS analyzed the data, and drafted the manuscript. All co-authors read and critically revised successive draft of the manuscript. All authors read and approved the final manuscript.

\section{Acknowledgments}

The authors acknowledge the in-kind support for data collection by Delvinia.

\section{References}

1. Shigemura J, Ursano RJ, Morganstein JC, Kurosawa M, Benedek DM. Public responses to the novel 2019 coronavirus (2019-nCoV) in Japan: Mental health consequences and target populations. Psychiatry Clin Neurosci. 2020;74(4):281-2.

2. Brooks SK, Webster RK, Smith LE, Woodland L, Wessely S, Greenberg N, et al. The psychological impact of quarantine and how to reduce it: rapid review of the evidence. The Lancet. 2020;395(10227):912-20.

3. Pfefferbaum B, North CS. Mental Health and the Covid-19 Pandemic. New England Journal of Medicine. 2020;383(6):510-2. 
4. Domènech-Abella J, Mundó J, Haro JM, Rubio-Valera M. Anxiety, depression, loneliness and social network in the elderly: Longitudinal associations from The Irish Longitudinal Study on Ageing (TILDA). Journal of Affective Disorders. 2019;246:82-8.

5. Calati R, Ferrari C, Brittner M, Oasi O, Olié E, Carvalho AF, et al. Suicidal thoughts and behaviors and social isolation: A narrative review of the literature. J Affect Disord. 2019;245:653-67.

6. Cacioppo JT, Hughes ME, Waite LJ, Hawkley LC, Thisted RA. Loneliness as a specific risk factor for depressive symptoms: cross-sectional and longitudinal analyses. Psychol Aging. 2006;21(1):140-51.

7. Rajkumar RP. COVID-19 and mental health: A review of the existing literature. Asian journal of psychiatry. 2020;52:102066-

8. Xiong J, Lipsitz O, Nasri F, Lui LMW, Gill H, Phan L, et al. Impact of COVID-19 pandemic on mental health in the general population: A systematic review. Journal of affective disorders. 2020;277:55-64.

9. Kessler RC, Merikangas KR, Wang PS. Prevalence, Comorbidity, and Service Utilization for Mood Disorders in the United States at the Beginning of the Twenty-first Century. Annual Review of Clinical Psychology. 2007;3(1):137-58.

10. Gao K, Wang Z, Chen J, Kemp DE, Chan PK, Conroy CM, et al. Should an assessment of Axis I comorbidity be included in the initial diagnostic assessment of mood disorders? Role of QIDS-16-SR total score in predicting number of Axis I comorbidity. Journal of Affective Disorders. 2013;148(2):256-64.

11. Lamers F, van Oppen P, Comijs HC, Smit JH, Spinhoven P, van Balkom AJ, et al. Comorbidity patterns of anxiety and depressive disorders in a large cohort study: the Netherlands Study of Depression and Anxiety (NESDA). J Clin Psychiatry. 2011;72(3):341-8.

12. Santini ZI, Jose PE, York Cornwell E, Koyanagi A, Nielsen L, Hinrichsen C, et al. Social disconnectedness, perceived isolation, and symptoms of depression and anxiety among older Americans (NSHAP): a longitudinal mediation analysis. The Lancet Public Health. 2020;5(1):e62-e70.

13. Shrira A, Hoffman Y, Bodner E, Palgi Y. COVID-19-Related Loneliness and Psychiatric Symptoms Among Older Adults: The Buffering Role of Subjective Age. Am J Geriatr Psychiatry. 2020;28(11):1200-4.

14. Palgi Y, Shrira A, Ring L, Bodner E, Avidor S, Bergman Y, et al. The loneliness pandemic: Loneliness and other concomitants of depression, anxiety and their comorbidity during the COVID-19 outbreak. Journal of affective disorders. 2020;275:109-11.

15. de Graaf R, Bijl RV, Spijker J, Beekman ATF, Vollebergh WAM. Temporal sequencing of lifetime mood disorders in relation to comorbid anxiety and substance use disorders. Social Psychiatry and Psychiatric Epidemiology. 2003;38(1):1-11.

16. Almeida OP, Draper B, Pirkis J, Snowdon J, Lautenschlager NT, Byrne G, et al. Anxiety, depression, and comorbid anxiety and depression: risk factors and outcome over two years. Int Psychogeriatr. 2012;24(10):1622-32.

17. Collins L, Lanza ST. Latent Class and Latent Transition Analysis. Hoboken, NJ: Wiley; 2010.

18. Petersen KJ, Qualter P, Humphrey N. The Application of Latent Class Analysis for Investigating Population Child Mental Health: A Systematic Review. Frontiers in Psychology. 2019;10(1214).

19. Fortin M, Lapointe L, Hudon C, Vanasse A, Ntetu AL, Maltais D. Multimorbidity and quality of life in primary care: a systematic review. Health Qual Life Outcomes. 2004;2:51.

20. Makovski TT, Schmitz S, Zeegers MP, Stranges S, van den Akker M. Multimorbidity and quality of life: Systematic literature review and meta-analysis. Ageing Res Rev. 2019;53:100903.

21. Johannessen EL, Andersson HW, Bjørngaard JH, Pape K. Anxiety and depression symptoms and alcohol use among adolescents - a cross sectional study of Norwegian secondary school students. BMC Public Health. 2017;17(1):494.

22. Rodgers $B$, Korten AE, Jorm AF, Jacomb PA, Christensen $H$, Henderson AS. Non-linear relationships in associations of depression and anxiety with alcohol use. Psychol Med. 2000;30(2):421-32.

23. Capasso A, Jones AM, Ali SH, Foreman J, Tozan Y, DiClemente RJ. Increased alcohol use during the COVID-19 pandemic: The effect of mental health and age in a cross-sectional sample of social media users in the U.S. Preventive Medicine. 2021;145:106422.

24. Danielsson A-K, Lundin A, Agardh E, Allebeck P, Forsell Y. Cannabis use, depression and anxiety: A 3-year prospective population-based study. Journal of Affective Disorders. 2016;193:103-8.

25. Cornelius JR, Kirisci L, Reynolds M, Clark DB, Hayes J, Tarter R. PTSD contributes to teen and young adult cannabis use disorders. Addictive Behaviors. 2010;35(2):91-4.

26. Cheung JTW, Mann RE, lalomiteanu A, Stoduto G, Chan V, Ala-Leppilampi K, et al. Anxiety and Mood Disorders and Cannabis Use. The American Journal of Drug and Alcohol Abuse. 2010;36(2):118-22.

27. Vanderbruggen N, Matthys F, Van Laere S, Zeeuws D, Santermans L, Van den Ameele S, et al. Self-Reported Alcohol, Tobacco, and Cannabis Use during COVID-19 Lockdown Measures: Results from a Web-Based Survey. European Addiction Research. 2020.

28. America is Drinking Its Way through the Coronavirus Crisis-That Means More Health Woes Ahead [press release]. 2020.

29. Finlay I, Gilmore I. Covid-19 and alcohol-a dangerous cocktail. BMJ. 2020;369:m1987.

30. Ahmed MZ, Ahmed O, Aibao Z, Hanbin S, Siyu L, Ahmad A. Epidemic of COVID-19 in China and associated Psychological Problems. Asian Journal of Psychiatry. 2020;51:102092.

31. Bartel SJ, Sherry SB, Stewart SH. Self-isolation: A significant contributor to cannabis use during the COVID-19 pandemic. Subst Abus. $2020: 1-4$.

32. Imtiaz S, Wells S, Rehm J, Hamilton HA, Nigatu YT, Wickens CM, et al. Cannabis Use During the COVID-19 Pandemic in Canada: A Repeated Crosssectional Study. J Addict Med. 2020.

33. Taylor S. The psychology of pandemics: Preparing for the next global outbreak of infectious disease. Newcastle upon Tyne, United Kingdom: Cambridge Scholars Publishing; 2019. xvi, 158-xvi, p. 
34. Szabo G, Mandrekar P. A recent perspective on alcohol, immunity, and host defense. Alcohol Clin Exp Res. 2009;33(2):220-32.

35. Simet SM, Sisson JH. Alcohol's Effects on Lung Health and Immunity. Alcohol Res. 2015;37(2):199-208.

36. Szabo G, Saha B. Alcohol's Effect on Host Defense. Alcohol Res. 2015;37(2):159-70.

37. Cabral GA, Rogers TJ, Lichtman AH. Turning Over a New Leaf: Cannabinoid and Endocannabinoid Modulation of Immune Function. J Neuroimmune Pharmacol. 2015;10(2):193-203.

38. Greineisen WE, Turner H. Immunoactive effects of cannabinoids: considerations for the therapeutic use of cannabinoid receptor agonists and antagonists. Int Immunopharmacol. 2010;10(5):547-55.

39. Winhusen T, Theobald J, Kaelber DC, Lewis D. Regular cannabis use, with and without tobacco co-use, is associated with respiratory disease. Drug Alcohol Depend. 2019;204:107557.

40. Callegaro M, DiSogra C. Computing Response Metrics for Online Panels. Public Opinion Quarterly. 2008;72(5):1008-32.

41. Kroenke K, Spitzer RL, Williams JB, Monahan PO, Löwe B. Anxiety disorders in primary care: prevalence, impairment, comorbidity, and detection. Ann Intern Med. 2007;146(5):317-25.

42. Roy-Byrne P, Veitengruber JP, Bystritsky A, Edlund MJ, Sullivan G, Craske MG, et al. Brief intervention for anxiety in primary care patients. J Am Board Fam Med. 2009;22(2):175-86.

43. Kroenke K, Spitzer RL, Williams JBW, Löwe B. The Patient Health Questionnaire Somatic, Anxiety, and Depressive Symptom Scales: a systematic review. General Hospital Psychiatry. 2010;32(4):345-59.

44. Spitzer RL, Kroenke K, Williams JBW, Löwe B. A Brief Measure for Assessing Generalized Anxiety Disorder: The GAD-7. Archives of Internal Medicine. 2006;166(10):1092-7.

45. Elton-Marshall T, Wells S, Jankowicz D, Nigatu YT, Wickens CM, Rehm J, et al. Multiple COVID-19 Risk Factors Increase the Likelihood of Experiencing Anxiety Symptoms in Canada. The Canadian Journal of Psychiatry. 2020:0706743720949356.

46. Radloff LS. The CES-D Scale:A Self-Report Depression Scale for Research in the General Population. Applied Psychological Measurement. 1977;1(3):385401.

47. Bauer DJ, Curran PJ. Distributional Assumptions of Growth Mixture Models: Implications for Overextraction of Latent Trajectory Classes. Psychological Methods. 2003;8(3):338-63.

48. Neely-Barnes S. Latent Class Models in Social Work. Social Work Research. 2010;34(2):114-21.

49. Yang K, Banamah A. Quota Sampling as an Alternative to Probability Sampling? An Experimental Study. Sociological Research Online. 2014;19(1):56-66.

50. Forster JJ. Sample Surveys: Nonprobability Sampling. In: Smelser NJ, Baltes PB, editors. International Encyclopedia of the Social \& Behavioral Sciences. Oxford: Pergamon; 2001. p. 13467-70.

51. Cumming RG. Is probability sampling always better? A comparison of results from a quota and a probability sample survey. Community Health Stud. 1990;14(2):132-7.

52. Heinrich LM, Gullone E. The clinical significance of loneliness: A literature review. Clinical Psychology Review. 2006;26(6):695-718.

53. Reddy BV, Gupta A. Importance of effective communication during COVID-19 infodemic. J Family Med Prim Care. 2020;9(8):3793-6.

54. Dalton L, Rapa E, Stein A. Protecting the psychological health of children through effective communication about COVID-19. Lancet Child Adolesc Health. 2020;4(5):346-7.

55. Back A, Tulsky JA, Arnold RM. Communication Skills in the Age of COVID-19. Ann Intern Med. 2020;172(11):759-60.

56. Rodriguez LM, Litt DM, Stewart SH. Drinking to cope with the pandemic: The unique associations of COVID-19-related perceived threat and psychological distress to drinking behaviors in American men and women. Addictive behaviors. 2020;110:106532-

57. Sinha R. Chronic stress, drug use, and vulnerability to addiction. Annals of the New York Academy of Sciences. 2008;1141:105-30.

\section{Supplementary Files}

This is a list of supplementary files associated with this preprint. Click to download.

- COVIDWave1to6merged.dta

- STROBEchecklistv4crosssectionalBMC19.doc

- SupplementalMaterialsept2021.docx 